\title{
The untold story of the COVID-19 pandemic: perceptions and views towards social stigma and bullying in the shadow of COVID-19 illness in Jordan
}

\author{
Amal Akour ${ }^{1,2}$ (D) Suha A. AlMuhaissen ${ }^{3} \cdot$ Mohammad B. Nusair $^{4}$. \\ Ala'a B. Al-Tammemi ${ }^{5,6,7}$ • Nouf N. Mahmoud ${ }^{2} \cdot$ Sarah Jalouqa $^{3}$. \\ Maissa N. Alrawashdeh ${ }^{8}$
}

Received: 10 March 2021 / Accepted: 26 August 2021 / Published online: 27 September 2021

(c) The Author(s), under exclusive licence to Springer Nature Switzerland AG 2021

\begin{abstract}
Stigmatization towards COVID-19 patients can lead to negative outcomes like social exclusion and bullying, and it may hinder the willingness of people to undergo testing. This study aimed to measure and explore the perception of stigmatization and bullying towards COVID-19 patients in Jordan. This was a web-based cross-sectional survey. Participants were recruited from social media platforms employing a snowball convenience sampling. The perception of bullying, beliefs regarding social consequences of infection, views on measures towards violators of patients' privacy, and how to reduce the stigma were assessed by self-reported measures. 397 participants returned completed questionnaires. The majority of respondents believed that COVID-19 patients in Jordan are getting bullied $(n=255,64.3 \%)$ and over $80 \%$ believed that people enjoy sharing identities, or news about COVID-19 patients. Although most respondents had adequate knowledge regarding transmission/prevention of COVID-19, they believed that all or some of the COVID-19 patients practiced something wrong to get infected $(n=358,90.2 \%)$. Moreover, $86.9 \%$ of respondents reported that people in Jordan were crossing their lines with bullying behaviors towards COVID-19 patients. However, these negative views would not discourage most respondents to get tested and follow the government's instructions if they or any of their acquaintances were suspected to be infected. Our study sheds the light on a high degree of stigma and bullying of COVID-19 patients during the early stage of the pandemic in Jordan. Hence, there is a need to develop and implement effective anti-stigma/anti-bullying campaigns that refute the misperception, raise public knowledge about COVID-19, and spread encouraging messages.
\end{abstract}

Keywords COVID-19 pandemic $\cdot$ Stigma $\cdot$ Bullying $\cdot$ Jordan $\cdot$ Social reactions

Amal Akour

a.akour@ju.edu.jo

Extended author information available on the last page of the article 


\section{Introduction}

The Coronavirus Disease 2019 (COVID-19) has firstly struck Jordan on March 2,2020 , when a traveler who was on a trip to Italy was tested positive for SARSCoV-2 upon returning back (Al-Tammemi 2020; Khatatbeh 2020, 2021a). Since then, the Jordanian government and the general population are in an active fight against this globally emerging infectious disease. Jordan was among the first countries in the Eastern Mediterranean Region (EMR) that imposed stringent measures to mitigate the COVID-19 spread. These measures included the declaration of national defense law which enforced a total country lockdown, nationwide curfew and quarantine, suspension of various work sectors and industries, cessation of on-campus educational activities, districts isolation, banning intercity travel, banning social events and gatherings as well as contact tracing and community testing (Akour et al. 2020, 2021; Al-Tammemi et al. 2020; Jordan Ministry of Healh 2020). The aforementioned measures have led to a slow pace of pandemic spread in Jordan in the period of March to August 2020 compared to the surrounding countries in the region (Al-Tammemi et al. 2021). As an economically vulnerable country, the government started to relax many restrictions as a part of the country's exit strategy aiming to return to a full-functioning economy again since early June 2020.

The COVID-19 pandemic has not just caused physical harm by the infectious process itself but this crisis has also led to various emotional, behavioral, socioeconomic, and psychological impacts in different contexts and populations (Aljaberi et al. 2021; Alrawashdeh et al. 2021; Fares et al. 2021; Folayan et al. 2021; Garbóczy et al. 2021; Khatatbeh et al. 2021; Lima et al. 2020; Nicola et al. 2020; Rajkumar 2020; Ramadan et al. 2021). People are naturally concerned about their health status especially when it comes to a highly contagious disease such as the COVID-19 (Kolozsvári et al. 2021), hence, different unprecedented social reactions and behaviors might evolve as a result of cultural misperception in society (Al-Tammemi and Tarhini 2021; Lin 2020). The fear and stigma were among the noticed social reactions among different communities worldwide.

Stigma can be described as a "mark of disgrace" that labels an individual or group of individuals leading to social exclusion and this can occur in-person or virtually (Budhwani and Sun 2020; Ramaci et al. 2020). Stigmatization of a specific group of people based on ethnicity or sickness has been experienced in many previous outbreaks and epidemics, such as stigmatization of native Americans upon the outbreak of Hantavirus in the United States (US) back in 1993 which was then called "Navajo disease," stigmatization of Chinatown community upon Bubonic plague outbreak, stigmatization and discrimination against Asians during Severe Acute Respiratory Syndrome (SARS) outbreak in 2003, in addition to HIV/AIDS and Ebola-associated stigma (Bruns et al. 2020). A recent example of pandemic-related stigmatization is referring to SARS-CoV-2 as "Chinese virus" or "Wuhan virus" which was extensively noticed on social media platforms, resulting in an Anti-Chinese stigma due to the COVID-19 pandemic (Alsawalqa 
2021; Budhwani and Sun 2020; Misra et al. 2020). This raises a concern that the enemy of the COVID-19 pandemic is not only the virus but also the stigma, discrimination, bullying, and rumors associated with the overall crisis (Badrfam and Zandifar 2020).

Social stigma is created and disseminated in a community through social interactions whether in the forms of face-to-face or online communications. Concerning health, infectious diseases' stigma (such as in COVID-19) can lead to substantial psychological impacts and negative health consequences among stigmatized individuals (Misra et al. 2020; Ramaci et al. 2020). This results in an increase of people's suffering especially among those who catch the infection as well as avoidance of seeking medical help at early stages to stay away from being labeled as an "infectious person" by society (Bruns et al. 2020; Ramaci et al. 2020). Stigmatization in a health context could be multifactorial in origin. Numerous factors might influence sickness-related stigmatization including lack of sufficient levels of awareness and knowledge about the infectious process and disease spread, misperception of danger, as well as excessive anxiety and fear (Badrfam and Zandifar 2020; Lin 2020).

Bullying is defined as "intentional, repetitive, negative behavior that can manifest in many ways" (Kaltiala-Heino and Fröjd 2011). Bullying as a subgroup of aggressive behavior can be categorized into direct bullying (such as physical bullying) and indirect bullying (such as spreading rumors) based on its nature. Besides, it can be categorized into reactive bullying (such as angry response) or proactive bullying (for dominance or self-gains) based on its motivation (Kaltiala-Heino and Fröjd 2011). Bullying can be practiced in many different ways including verbal, physical, or even electronic means (cyber-bullying) (Kaltiala-Heino and Fröjd 2011). Therefore, and considering the importance of this topic which is under-investigated in the Middle East and North Africa (MENA) region amidst the COVID-19 pandemic, the present study aimed to assess and explore the perception of stigmatization and bullying towards COVID-19 patients in Jordan from the perspectives of individuals who did not contract the disease.

\section{Methodology}

\section{Study setting}

Our study was conducted in Jordan, a middle-income country located in the Eastern Mediterranean Region (EMR) with a population count of around 10.8 million (Department of Statistics - Jordan 2020). The official language of the country is Arabic. $93 \%$ of Jordanians are Muslims, $6 \%$ are Christians and the remaining $1 \%$ follow other religions. Jordan is also popular for its cultural diversity. 


\section{Study design, survey instrument, and data collection}

A cross-sectional design was utilized. Data for this project were collected using an anonymous web-based structured questionnaire. The data collection tool was developed by the authors based on extensive review of published relevant literature (Van Brakel 2006; Dowshen et al. 2009; Semrau et al. 2015), in addition to the authors' observation of the Jordanian society's reactions on social media platforms during the COVID-19 pandemic.

A 30-item questionnaire of four sections was developed to obtain data from the Jordanian population about how they deal with COVID-19 patients. In the initial section of the questionnaire, demographic information and descriptive data were collected, including age, gender, the governorate of residence/region, education, employment status, and profession. Another two sections have assessed multiple domains including the participants' general knowledge about the COVID19, participants' beliefs/attitudes towards the social consequences of contracting COVID-19, and the participants' views on the measures towards those who violate COVID-19 patients' privacy, those who put others at risk of getting infected, and on how to reduce stigma towards COVID-19 patients. Additionally, one section has assessed the participants' perception about bullying and stigma towards COVID-19 patients in Jordan. This section consisted of seven questions on a 5-point Likert scale ranging from 1 (strongly disagree) to 5 (strongly agree) and scored accordingly. Thus, the total score of a participant's views on bullying and stigma towards COVID-19 patients could range from 7-35. Accordingly, and for the aim of analyses, we divided the participants into two subgroups/categories based on their score: the low-bullying viewers' group (score range 7-21) and the high-bullying viewers' group (score range 22-35).

The questionnaire was evaluated for face and content validity by experts in the field of social sciences in collaboration with the research team. Afterward, a pilot test of the 30-item questionnaire was conducted on 20 participants who were randomly selected. The comments received from the pilot stage were evaluated and used to modify the data collection tool as needed before starting the main procedure of data collection. The pilot responses were not included in our final analyses.

Given the unfolding situation of the COVID-19 pandemic and the strict control measures during the period of conducting our study, the decision was made by the research team to collect the data using a web-based survey tool. Google Form ${ }^{\circledR}$ (A cloud-based survey tool) was utilized to construct the survey which was then administered in modern standard Arabic and was disseminated to the Jordanian community using various social media platforms (e.g., Facebook®, WhatsApp Messenger®, and LinkedIn $\left.{ }^{\circledR}\right)$. People were requested to share the survey questionnaire with their relatives and friends as possible; thus, employing a snowball convenience sampling strategy. Data collection was carried out in May-June 2020 for a period of four weeks. The questionnaire was supplemented with a cover letter and instructions 
to participants. Besides, individuals who were interested to participate in this study provided their informed consent (electronically) before responding to the questionnaire. The inclusion criteria were age $\geq 18$ years, capability of reading and understanding Arabic, did not contract COVID-19, and willingness to participate voluntarily. To minimize social desirability bias, assurance was given to participants that the data they provide will be confidential and their identity will stay anonymous. The collected data were encrypted, and securely stored, and further analyses were done anonymously.

\section{Statistical analyses}

The sample size was calculated using Open Source Epidemiologic Statistics for Public Health Version 3.01(Dean et al. 2013). A sample size of at least 385 participants was required, with $95 \%$ confidence level, 50\% response distribution and $5 \%$ margin of error. Categorical variables were described as a frequency counts and percentages, and continuous variables were reported as a mean and standard deviation (SD). Moreover, Chi-square test was employed to assess significant differences between high-bullying and low-bullying viewers' groups. A $p$ value $<0.05$ was implemented for statistical significance. Data analyses were performed using SPSS 25.0 (IBM, Armonk, NY).

\section{Ethical considerations}

The ethical approval for conducting our study was sought and obtained from the Institutional Review Board (IRB) at the Deanship of Academic Research-The University of Jordan (IRB Ref. 307/19). Besides, all methods were carried out following the national guidelines and conforming to the ethical standards of the Declaration of Helsinki. The questionnaire ensured the confidentiality and anonymity of study participants. All participants were informed about the study and electronic written informed consent was obtained before completing the survey.

\section{Results}

\section{Participants' sociodemographic characteristics}

A total of 397 participants have returned completed questionnaires. The majority were females $(n=278,70 \%)$, and the mean age of participants was 30.1 years (SD: 9.82). Most of respondents had a bachelor's degree $(n=270,68 \%)$, and $60.9 \%$ $(n=242)$ had non-medical professions. Moreover, 95\% of the participants live in the central and northern regions of Jordan where most of the COVID-19 cases were confirmed at the time of conducting this study (See Table 1). Of the overall sample, 
Table 1 Socio-demographic characteristics of respondents $(n=397)$

\begin{tabular}{llc}
\hline Variable & & $\mathrm{N}(\%)$ \\
\hline Gender & Male & $119(30 \%)$ \\
& Female & $278(70 \%)$ \\
Education & Up to secondary school & $8(2 \%)$ \\
& Diploma degree & $13(3.3 \%)$ \\
& Bachelor's degree & $270(68 \%)$ \\
Employment status & Post-graduate degree & $106(26.7 \%)$ \\
& Employed & $182(45.8 \%)$ \\
Profession & Unemployed & $215(54.2 \%)$ \\
& Health-related & $155(39 \%)$ \\
Geographical area & Non-health related & $242(61 \%)$ \\
& Northern region & $95(23.9 \%)$ \\
& Central region & $284(71.5 \%)$ \\
Age (mean \pm SD) & Southern region & $18(4.5 \%)$ \\
\hline
\end{tabular}

$22.2 \%$ reported that they knew someone who got infected by COVID-19, and of them $(n=56,63.3 \%)$ were bullied for contracting the disease.

\section{Participants' perception towards bullying during the COVID-19 pandemic}

In this domain, most participants believed that COVID-19 patients in Jordan were getting bullied and harassed $(n=255,64.3 \%)$. Besides, more than $80 \%$ of respondents believed that people in Jordan enjoy sharing identities, pictures, news about COVID-19 patients, and take patients' information as a joke they can spread in their social circles (Table 2). On the other hand, less than half of respondents believed that healthcare providers are taking good confidentiality measures and the Jordanian cybercrime authority was taking proper actions against those who violate COVID19 patients' privacy (Table 2). Furthermore, $86.9 \%$ of the participants reported that people in Jordan were crossing their lines with bullying behaviors towards COVID19 patients (Table 2). More details are provided in Table 2.

\section{Participants' knowledge about the COVID-19 illness, and their beliefs regarding social consequences of contracting COVID-19}

Based on the scoring and categorization described in data analysis section, of the 397 participants, around 31.2\% $(n=124)$ believed that COVID-19 patients were getting highly bullied (i.e., high-bullying viewers group) while the remaining ( $n=273$, $68.8 \%$ ) of participants believed that COVID-19 patients were getting no to little bullying (i.e., low-bullying viewers group). 


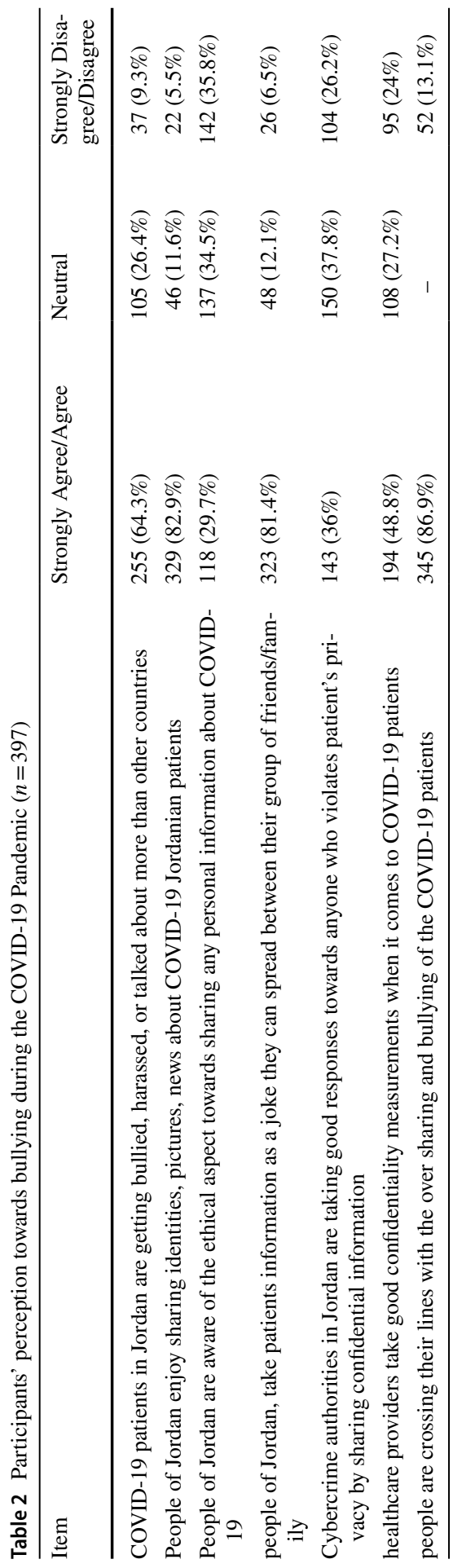




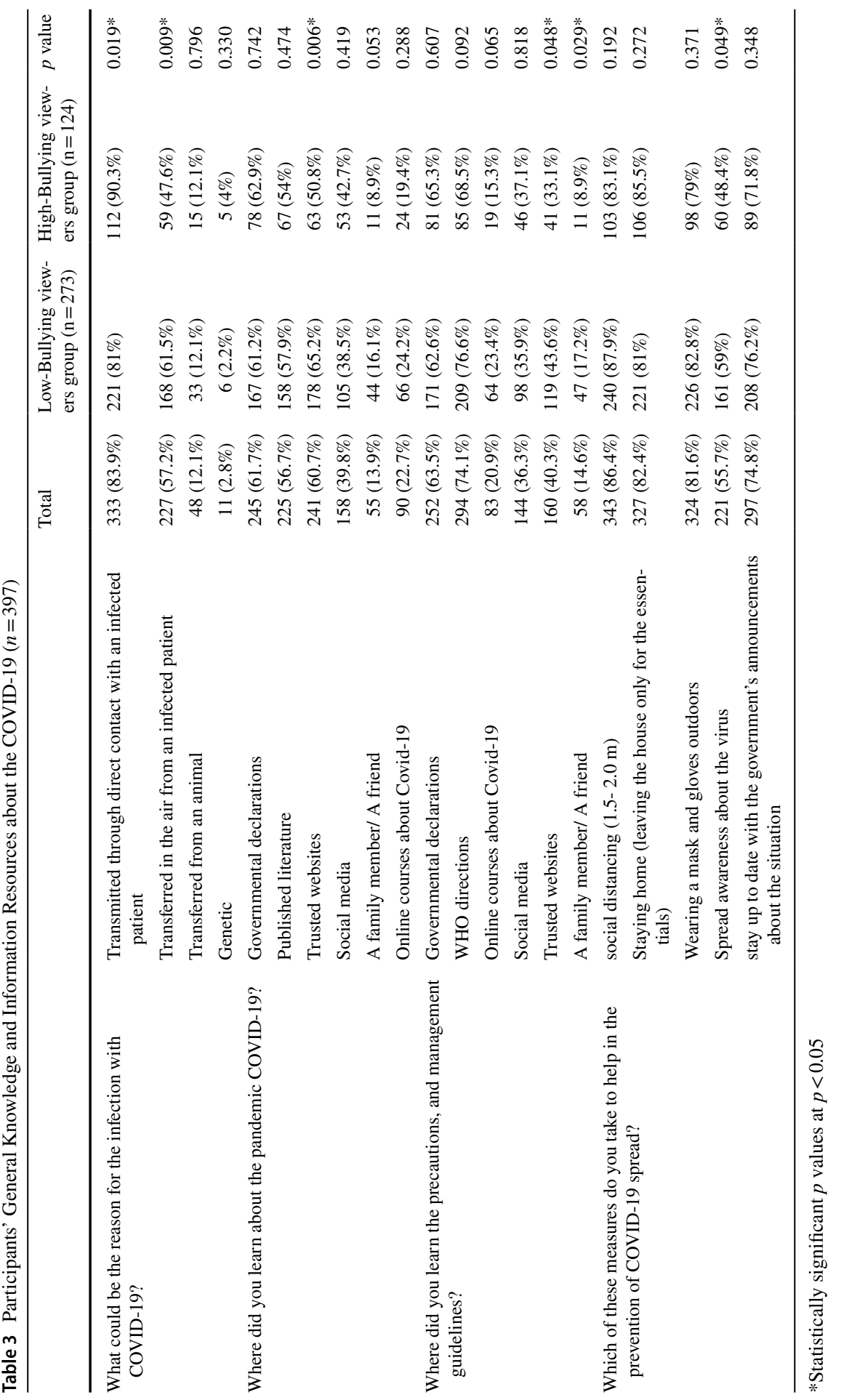


Overall, the majority of respondents had adequate knowledge regarding the transmission routes of COVID-19 and its prevention measures. However, the highbullying viewers' group had significantly higher knowledge about virus transmission $(p=0.019)$. On the other hand, the low-bullying viewers' group believed that spreading awareness about the virus would be an effective measure to prevent the viral spread, and this was significantly higher than of the high-bullying viewers' group $(p=0.049)$. Also, the majority of participants learned about the pandemic news, precautions, and management guidelines from governmental declarations and WHO directions. Interestingly, the low-bullying viewers' group relied on trusted websites and family members or friends more than the high-bullying viewers' group ( $p=0.006$ and 0.029 , respectively). See Table 3 for more details.

Most participants $(n=358,90.2 \%)$ believed that all or some of COVID19 patients did something wrong, so they consequently contracted the infected (Table 4). As a result, nearly half of respondents believed that COVID-19 patients will be socially impacted and $32 \%$ of respondents believed that these patients would lose their jobs. The high-bullying viewers' group believed that COVID-19 patients will be denied education and have lesser chances to get employed significantly more than the low-bullying viewers' group ( $p=0.027$ and $p=0.004$ respectively; Table 4 ). These negative views towards COVID-19 patients would not discourage the majority of respondents to get tested and follow government instructions if they or any of their acquaintances were suspected to be infected (Table 4). However, a handful of respondents believed that COVID-19 is not a serious virus, and it can be dealt with as any other seasonal flu and this belief was significantly higher in the high-bullying viewers' group $(p=0.04)$. 


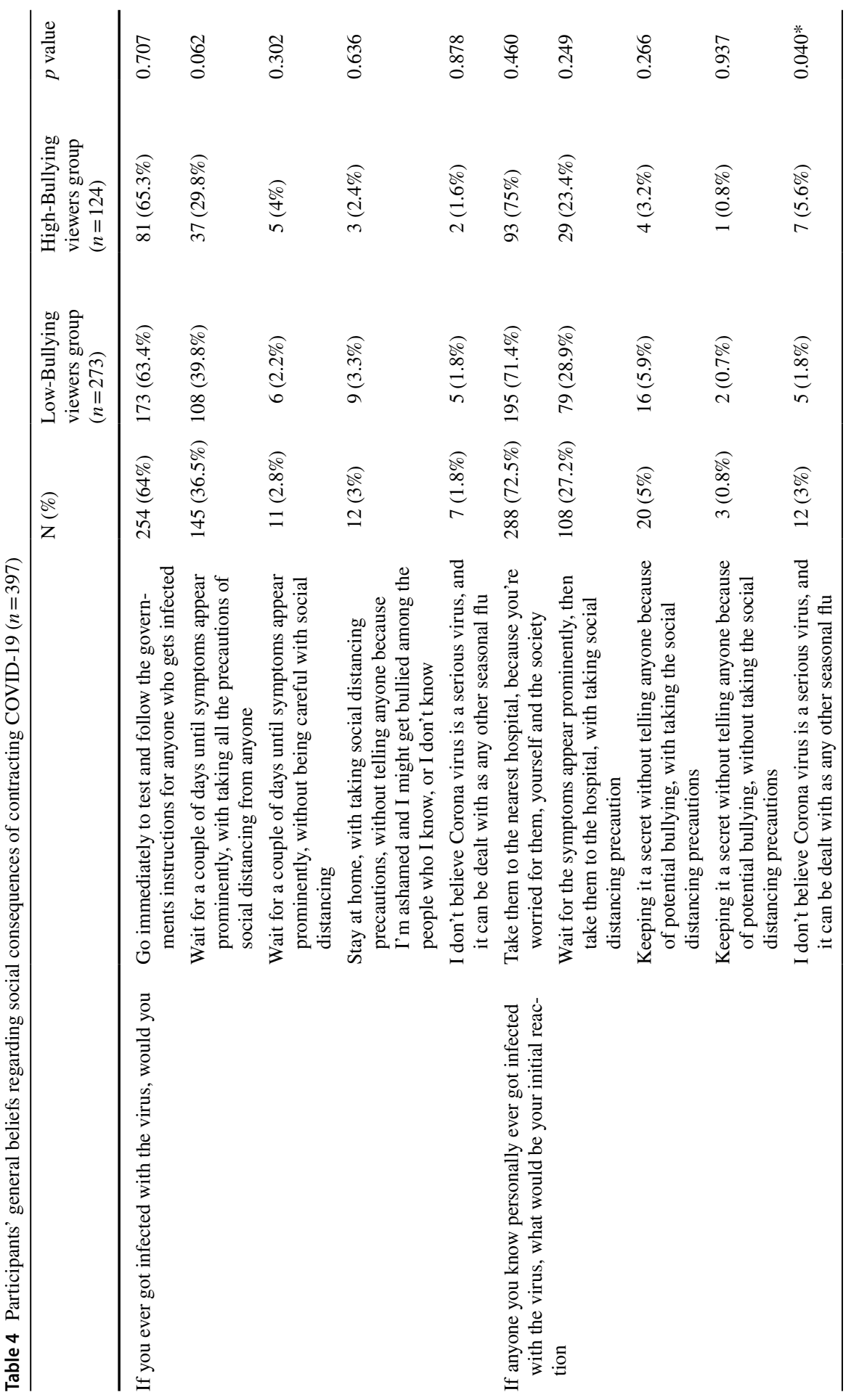




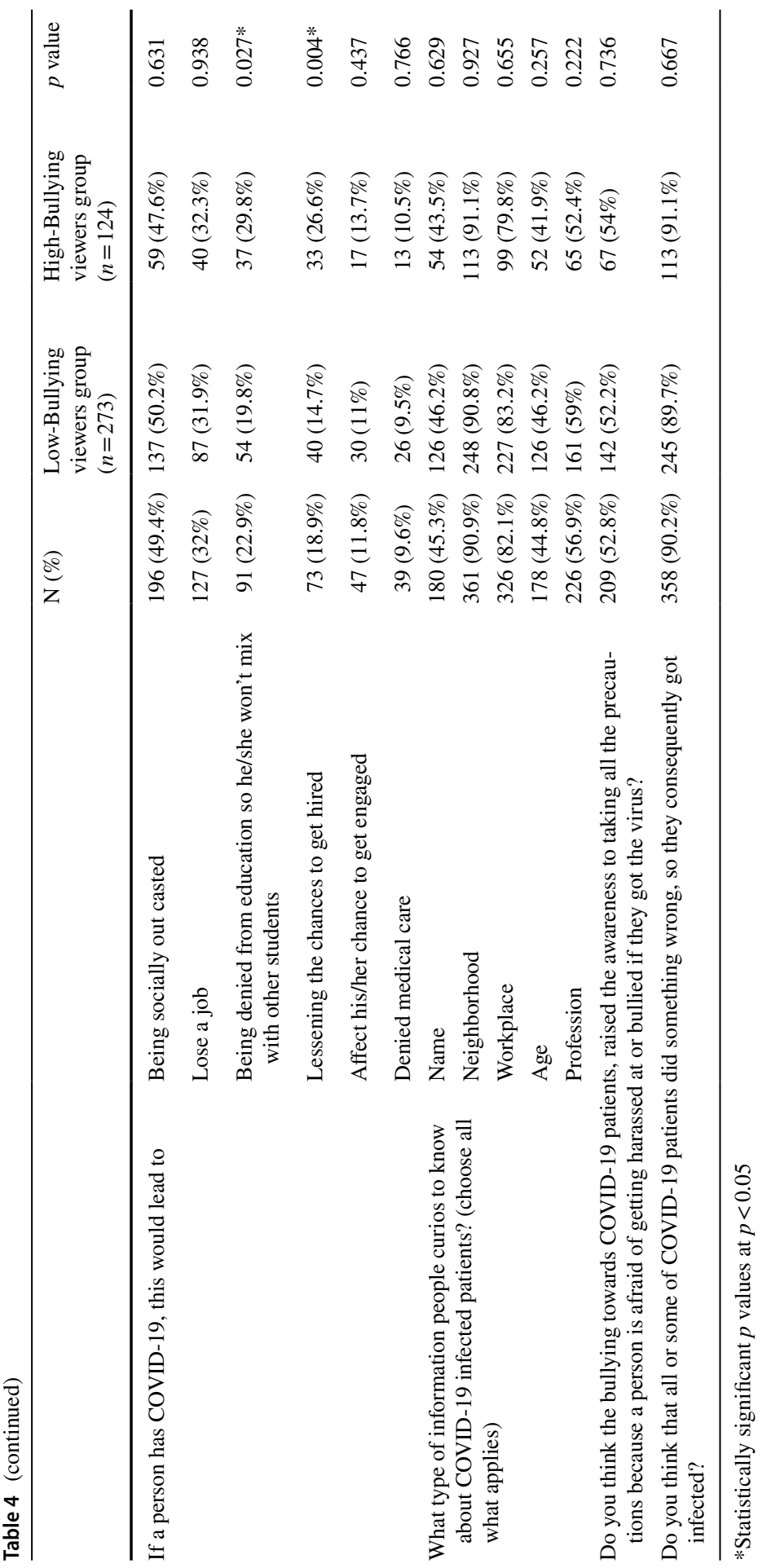




\section{Views on anti-stigma/anti-bullying measures during the COVID-19 pandemic}

In this domain, participants were asked to report their views regarding anti-stigma/ anti-bullying measures towards those who violate patients' privacy, or infected persons who put others at risk of getting infected, and on how to reduce stigma towards COVID-19 patients. Most participants reported that those who do not take good confidentiality measures or violate COVID-19 patients' privacy should be legally punished by paying a fine $(n=277,69.8 \%)$ or imprisonment $(n=124,31.2 \%)$, or both $(n=104,26.2 \%)$ (Fig. 1). However, a minority of participants $(n=26,6.5 \%)$ reported that those who share falsified information or share COVID-19 patients' private information should be fired from their jobs (Fig. 1). On the other hand, most participants reported that infected persons who know that they are infectious and still socialize with relatives and friends without telling them/without isolation should be also punished by paying a fine $(n=217,54.7 \%)$, or should be both fined and imprisoned ( $n=193,48.6 \%)$. See Fig. 1 for more illustrations.

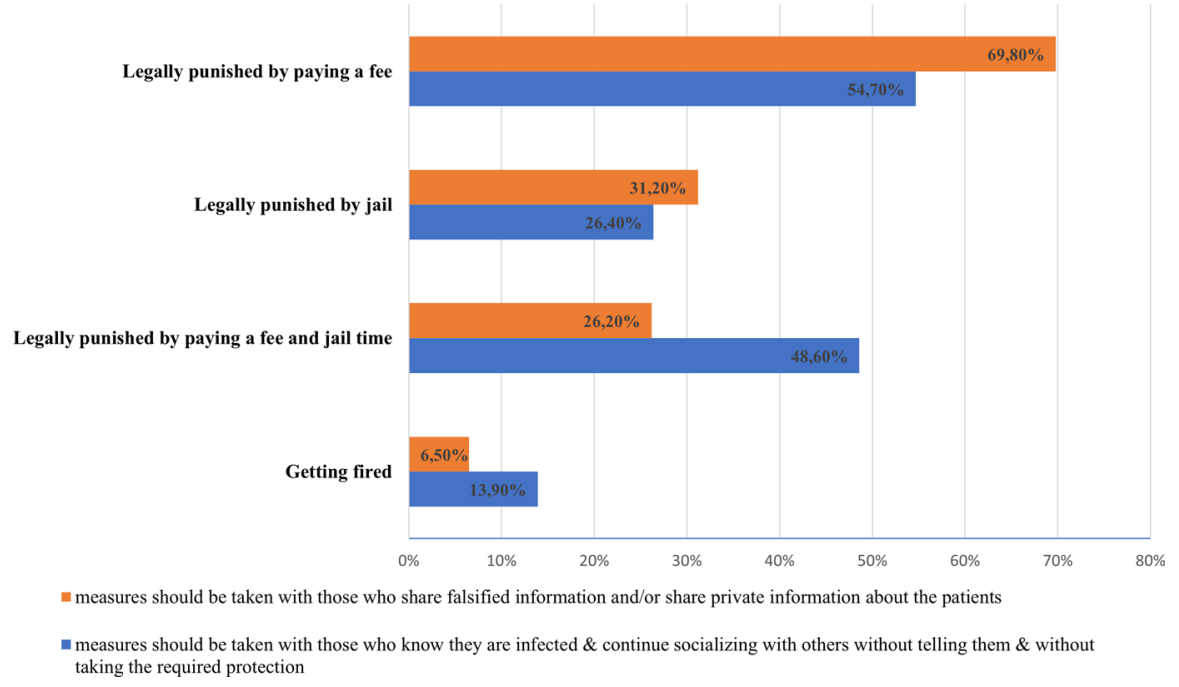

Fig. 1 Participants' views on the measures towards those who violate COVID-19 patients' privacy or those who put others at risk of getting infected

Regarding the participants views on how to reduce stigma and bullying towards COVID-19 patients, most respondents $(n=334,84.1 \%)$ reported that these bullying behaviors can be reduced by raising the awareness regarding COVID-19, followed by declaring that there is a chance of contracting the disease despite observing precautionary measures $(n=321,80.9 \%)$, behaving more ethically $(n=301,75.8 \%)$, or being aware that asymptomatic infections are a possibility; thus, someone around could be infected without knowing that $(n=289,72.8 \%)$ (Fig. 2). The low-bullying viewers group had significantly stronger beliefs that bullying can be reduced by clarifying the fact that there is a chance of getting infected despite all protection 


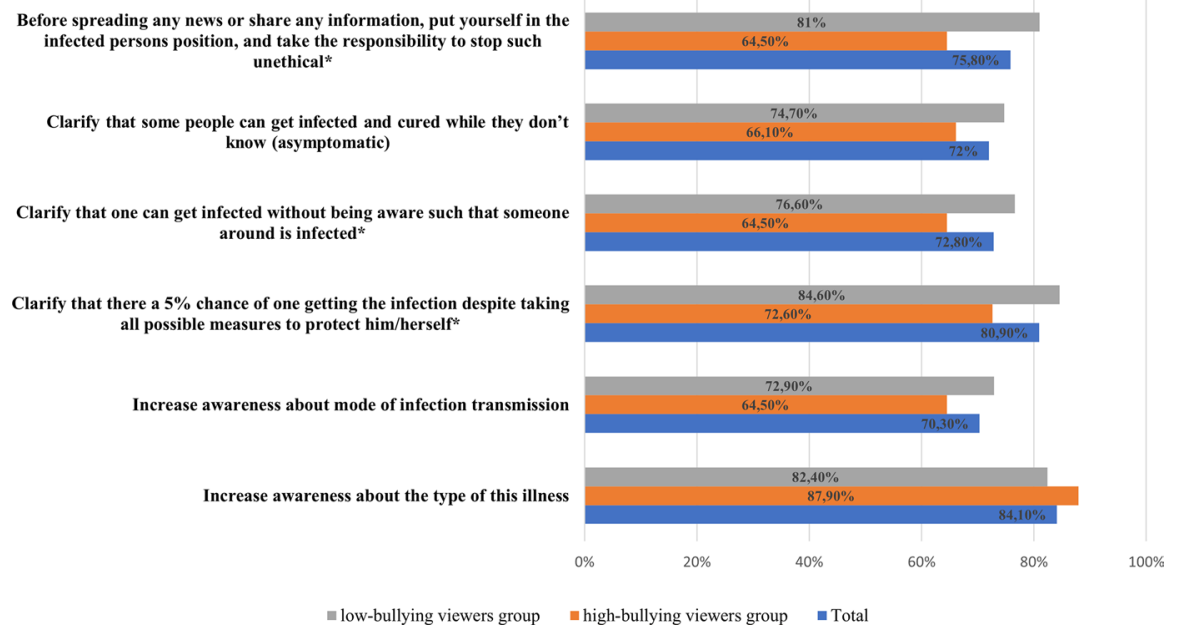

Fig. 2 Participants' views on how to reduce stigma and harassment towards COVID-19 patients. (Statistically significant items at $p<0.05$ are marked with asterisk)

measures taken by someone and by being more considerate of others' situation (items marked with asterisk, $p<0.05$; Fig. 2). More illustrations are described in Fig. 2.

\section{Discussion}

Social stigma in the context of health and illness is considered a serious global public health issue. This stigma can lead to a repetition of negative actions (physical, verbal, or psychological) specifically directed towards ill people over time, which is known as bullying (Pantelic et al. 2017). Stigmatization of patients or others who share specific characteristics of health conditions is most widely studied in several common diseases such as HIV/AIDS, mental illness, inflammatory bowel diseases, autoimmune diseases, and others (Chambers et al. 2015; Dibley et al. 2018; Kalantari et al. 2018; Kaushik et al. 2016). Stigmatization is usually increasing in outbreaks where the patients are labeled, stereotyped, discriminated against, and treated separately. Such treatments may undermine the patients' social and health behaviors and are associated with increased social anxiety and shame; moreover, stigma can also negatively impact the patients' families and communities. Stigma related to infectious diseases, in particular, prevents efficient control of the conditions and keeps ongoing transmission of infection (Fischer et al. 2019). Infectious disease-related stigma among many other confidentiality and financial challenges can act as a barrier that might hinder people from getting tested or seeking medical help as reported in the literature (Egelko et al. 2020; Ferree et al. 2021; Taylor et al. 2020; Teksin et al. 2020).

It is worth mentioning that stigma towards any new outbreaks such as the current COVID-19 is related to the fact that the disease is new and correlated with many unknown aspects, especially health outcomes and possible complications. 
In this study, stigmatization of patients with COVID-19 in Jordan has been explored in terms of three main domains: Knowledge about the disease, perception of the bullying behaviors towards COVID-19 patients, and understanding the measures of how to reduce COVID-19-related stigma. The obtained results point out that the majority of participants believed that patients with COVID-19 were exposed to humiliation and bullying, and they were used as an attractive material for spreading jokes. This behavior could be considered as a possible stress-coping mechanism to the pandemics. It was reported in the literature that the COVID19 pandemic may paly a potential role in contributing to bullying behaviors at various contexts including healthcare settings (Yahya et al. 2020). Bullying-based practices and harassment towards patients may be utilized by some individuals in societies to reduce the impact of stress and anxiety related to novel and unknown pandemics. A real-life example of such behavior in Jordan during the last quarantine was when jokes and memes erupted as bubbles and circulated rapidly on social media regarding the suspicion of an individual with COVID-19 who "fled" from mandatory quarantine. Additionally, Dye et al. (2020) pointed to a significant COVID-19-related bullying among healthcare workers, including those who treat COVID-19 patients, and this was characterized as a social crisis embedded within the pandemic (Dye et al. 2020). Besides, in Dye et al.'s study, many participants have witnessed or experienced COVID-19-related bullying and stigmatization during their daily activities in public places. These aggressive behaviors may target people who are suspected to have COVID-19, infected patients, and even those who do not follow precautionary measures like physical distancing in public places. During the Ebola pandemic in Africa, stigmatization and discrimination were obvious and continued among the survivors too (Fischer et al. 2019).

Similarly, bullying, attacks, and hate crimes increased in the US towards Asians and Asian Americans due to the COVID-19 global outbreak. A recent study indicated the rise in referencing the novel COVID-19 virus as the "Chinese virus" on Twitter as a form of COVID-19 stigma (Budhwani and Sun 2020). In Jordan, a recent study has assessed cyber-bullying in the shadow of COVID-19, and found that East and Southeast Asian students have experienced social stigmatization and bullying due to the connection between their countries of origin and the COVID-19 (Alsawalqa 2021). The study also described serious psychological and social impacts among stigmatized victims. Social stigma is associated with other diseases too; Dipley et al. have demonstrated how stigmatization of patients with inflammatory bowel diseases is profoundly affecting the ability of patients to adjust and control the disease and enhancing the development of depressive symptoms (Dibley et al. 2018; Roberts et al. 2020).

Our results indicate that most respondents do not have much trust in the confidentiality actions and procedures taken by the healthcare providers or the cybercrime authorities in protecting the COVID-19 patients' privacy. They believe in the urgent need to take strict and real measures against violation of COVID-19 patients' privacy. Lack of efficient governmental actions and global policy regarding COVID-19 patients' privacy may fuel the bullying behaviors and harassment actions and reduce the effectiveness of any efforts to address the disease stigma (Ransing et al. 2020). 
China and other countries took several measures and set standards to strengthen the protection of COVID-19 patients' privacy (ReedSmith 2020).

Interestingly, there is a correlation between the level of the respondents' knowledge of COVID-19 transmission methods and their beliefs regarding the level of bullying that COVID-19 patients are exposed to. Stigma is usually associated with a lack of knowledge about the spreading and transmission of diseases and a need to blame someone. In our study, the high-bullying viewers' group had significantly higher awareness about the virus transmission than the low-bullying viewers' group. Such high knowledge may help respondents to identify and perceive the bullying attitude towards COVID-19 patients. A recent study conducted in Egypt demonstrated that knowledge of COVID-19 positively correlated with the attitude towards COVID-19 as a preventable disease (Abdel Wahed et al. 2020). Recent reports indicate that efforts towards public education about the disease and the rationale for quarantine can significantly reduce stigmatization (Bruns et al. 2020).

Fortunately, the results point out that the official declaration and WHO directions are considered the primary resources for most respondents regarding the pandemic COVID-19 news, precautions, and management guidelines. However, the low-bullying viewers' group relies on trusted websites and family members or friends significantly higher than the high-bullying viewers' group, which may justify their low perception of COVID-19 patients' bullying.

Furthermore, the results demonstrate that most of the respondents believe that COVID-19 patients are responsible for getting infected with the virus. This belief may also justify the observed negative views and thoughts towards COVID-19 patients. Besides, the lack of efficient education concerning the transmission of the virus and its incubation period can fuel stigmatization and enrich such beliefs regarding how and why COVID-19 patients are getting infected. Therefore, those who demonstrated greater susceptibility towards bullying of COVID-19 patients (High bullying group), expressed more negative views and fears towards COVID-19 patients in terms of social acceptance, education, and employment.

While our study is among the limited literature that investigated the extent of stigma and bullying towards COVID-19 patients, there are some noteworthy limitations that should be acknowledged, including the use of convenience sampling strategy which affects the representativeness of our sample and the generalizability of the results. However, this was believed to be the most feasible strategy of sampling amid the COVID-19 crisis. Besides, our study was conducted among those who did not contract the disease, thus, it lacks the perspectives of patients and their views/experiences. This was attributed to the fact that accessing COVID-19 patients was difficult during the period of conducting our study and the high level of stigmatization of patients noticed on social media platforms which may affect their willingness to participate in such research. Nevertheless, we encourage for more inclusive studies that explore the side of the untold story from the side of COVID-19 patients in general or stigmatized victims. Moreover, most participants in our study were females, and we might have missed portions of the target study population that include illiterate people, people with limited digital literacy as well as non-Arabic speaking expatriates in the country. 


\section{Conclusion}

Our study sheds the light on a very important, yet neglected, issue of stigma and bullying of COVID-19 patients. Most of our study participants believed that COVID-19 patients were bullied, and that the Jordanian community enjoys shaming them by spreading jokes and their news. More than $90 \%$ believed the patients did something wrong and got infected, therefore this could impact patients or excluding them from job or education opportunities. Hence, there is a need to develop and implement effective anti-stigma/anti-bullying campaigns that refute the misperception about COVID-19, increase the public's knowledge about COVID-19, and spreads encouraging and sympathetic messages. The latter could be done utilizing various social media platforms, given the high acceptability and access rate. These strategies are expected to lower the fear and stigma. Future studies should assess the effect of such programs on stigma and bullying potentials towards COVID-19 patients in society.

Acknowledgements The authors provide their sincere appreciation to the study participants.

Author Contributions Conceptualization and Methodology: AA, SAA, SJ, NNM, MNA, and ABA; Data curation: AA, SAA, and SJ; Statistical Analysis, Validation, and Interpretation: MBN; Writing-Original draft preparation: AA, SAA, MBN, ABA, SJ, NNM, and MNA; Writing-Reviewing and Editing: AA, ABA, and SAA.

Funding This research project did not receive any specific grant from funding agencies in the public, commercial, or not-for-profit sectors.

Data Availability The dataset generated and analyzed in this study is available from the corresponding author on a reasonable request.

\section{Declarations}

Conflict of interest The authors have no conflicts of interest to declare that are relevant to the content of this article.

Ethical approval The ethical approval for conducting our study was sought and obtained from the Institutional Review Board (IRB) at the Deanship of Academic Research-The University of Jordan (IRB Ref. 307/19). Besides, all methods were carried out following the national guidelines and conforming to the ethical standards of the Declaration of Helsinki. The questionnaire ensured the confidentiality and anonymity of study participants. All participants were informed about the study and electronic written informed consent was obtained before completing the survey.

Informed consent Informed consent was obtained from all participants included in the study.

\section{References}

Akour A, Al-Tammemi AB, Barakat M, Kanj R, Fakhouri HN, Malkawi A, Musleh G (2020) The impact of the COVID-19 pandemic and emergency distance teaching on the psychological status of university teachers: a cross-sectional study in Jordan. Am J Trop Med Hyg 103(6):2391-2399

Akour A, Elayeh E, Tubeileh R, Hammad A, Ya'Acoub R, Al-Tammemi AB (2021) Role of community pharmacists in medication management during COVID-19 Lockdown. Pathogens and Global Health 115:1-10 
Al-Tammemi AB (2020) The battle against COVID-19 in Jordan : an early overview of the jordanian experience. Front Public Health 8:188

Al-Tammemi AB, Akour A, Alfalah L (2020) Is it just about physical health? An online cross-sectional study exploring the psychological distress among university students in Jordan in the midst of COVID-19 pandemic. Front Psychol 11:562213

Al-Tammemi AB, Tarhini Z (2021) Beyond equity: advocating theory-based health promotion in Parallel with COVID-19 mass vaccination campaigns. Public Health in Practice 2:100142

A1-Tammemi AB, Tarhini Z, Akour A (2021) A swaying between successive pandemic waves and pandemic fatigue: Where does Jordan stand? Annals of Medicine and Surgery 65:102298

Aljaberi MA, Alareqe NA, Qasem MA, Alsalahi A, Noman S, Ibrahim MIM (2021) Rasch modeling and multilevel confirmatory factor analysis for the usability of the impact of event scale-revised (IES-R) during the COVID-19 pandemic. SSRN PrePrints. https://doi.org/10.2139/ssrn.3815681

Alrawashdeh HM, Al-Tammemi AB, Alzawahreh MK, Al-Tamimi A, Elkholy M, Al Sarireh F, Abusamak M, Elehamer NM, Malkawi A, Al-Dolat W, Abu-Ismail L, Al-Far A (2021) Occupational burnout and job satisfaction among physicians in times of COVID-19 crisis: a convergent parallel mixedmethod study. BMC Public Health 21:811

Alsawalqa RO (2021) Cyberbullying, social stigma, and self-esteem: the impact of COVID-19 on students from East and Southeast Asia at the university of Jordan. Heliyon 7(4):e06711

Badrfam R, Zandifar A (2020) Stigma Over COVID-19; new conception beyond individual sense. Arch Med Res 51(6):593-594

Brakel V, Wim H (2006) Measuring health-related stigma-a literature review. Psychol Health Med 11(3):307-334

Bruns DP, Kraguljac NV, Bruns TR (2020) COVID-19: facts, cultural considerations, and risk of stigmatization. J Transcult Nurs 31(4):326-332

Budhwani H, Sun R (2020) Creating COVID-19 stigma by referencing the novel coronavirus as the 'Chinese Virus' on Twitter: quantitative analysis of social media data. J Med Internet Res 22(5):19301

Chambers LA, Sergio Rueda D, Baker N, Wilson MG, Deutsch R, Raeifar E, Rourke SB (2015) Stigma, HIV and Health: a qualitative synthesis. BMC Public Health 15:848

Dean, Andrew G., Kevin M. Sullivan, and Minn Minn Soe. 2013. “OpenEpi: Open Source Epidemiologic Statistics for Public Health."

Department of Statistics - Jordan. 2020. "Population Count." [Online]. Retrieved April 16, 2020 (http:// dosweb.dos.gov.jo/).

Dibley L, Norton C, Whitehead E (2018) the experience of stigma in inflammatory bowel disease: an interpretive (hermeneutic) phenomenological Study. J Adv Nurs 74(4):838-851

Dowshen N, Binns HJ, Garofalo R (2009) Experiences of HIV-related stigma among young men who have sex with men. AIDS Patient Care STDS 23(5):371-376

Dye TD, Alcantara L, Siddiqi S, Barbosu M, Sharma S, Panko T, Pressman E (2020) Risk of COVID19-related bullying, harassment and stigma among healthcare workers: an analytical cross-sectional global study. BMJ Open 10(12):046620

Egelko A, Arnaout L, Garoon J, Streed C, Berger Z (2020) 'Do I Have to Be Tested?': Understanding reluctance to be screened for COVID-19. Am J Public Health 110(12):1769-1771

Fares, ZEA., Al-Tammemi AB, Gadelrab HF, Lin C-Y, Aljaberi MA, Alhuwailah A, Roubi ML (2021) "Arabic COVID-19 Psychological Distress Scale: Development and Initial Validation." BMJ Open 11(6): 046006.

Ferree, Karen E., Adam S. Harris, Boniface Dulani, Kristen Kao, Ellen Lust, and Erica Metheney. 2021. "Stigma, Trust, and Procedural Integrity: COVID-19 Testing in Malawi." World Development 141:105351.

Fischer LS, Mansergh G, Lynch J, Santibanez S (2019) Addressing disease-related stigma during infectious disease outbreaks. Disaster Med Public Health Prep 13(5-6):989-994

Folayan MO, Ibigbami O, El Tantawi M, Brown B, Aly NM, Ezechi O et al (2021) Factors associated with financial security food security and quality of daily lives of residents in Nigeria during the first wave of the COVID-19 pandemic. Int J Environ Res Public Health 18(15):7925. https://doi.org/10. 3390/ijerph18157925

Garbóczy S, Szemán-Nagy A, Ahmad MS, Harsányi S, Ocsenás D, Rekenyi V, Al-Tammemi AB, Kolozsvári LR (2021) Health Anxiety, perceived stress, and coping styles in the shadow of the COVID-19. BMC Psychology 9(1):53

Jordan Ministry of Healh. 2020. "Jordan COVID-19 Platform.” [Online]. Retrieved October 25, 2020 (https://corona.moh.gov.jo/en). 
Kalantari S, Karbakhsh M, Kamiab Z, Kalantari Z, Sahraian MA (2018) Perceived social stigma in patients with multiple sclerosis: a study from Iran. Acta Neurol Taiwan 27(1):1-8

Kaltiala-Heino R (2011) Correlation between bullying and clinical depression in adolescent patients. Adolesc Health Med Ther 37:1

Kaushik A, Kostaki E, Kyriakopoulos M (2016) The stigma of mental illness in children and adolescents: a systematic review. Psychiatry Res 243:469-494

Khatatbeh M (2020) Efficacy of Nationwide Curfew to encounter spread of COVID-19: a case from Jordan. Front Public Health 8:394

Khatatbeh M (2021) The battle against COVID-19 in Jordan: from extreme victory to extreme burden. Front Public Health 8:1038

Khatatbeh M, Khasawneh A, Hussein H, Altahat O, Alhalaiqa F (2021a) Psychological Impact of COVID-19 pandemic among the general population in Jordan. Front Psych 12:618993

Khatatbeh M, Alhalaiqa F, Khasawneh A, Al-Tammemi AB, Khatatbeh H, Alhassoun S, Al Omari O (2021b) The experiences of nurses and physicians caring for COVID-19 patients: findings from an exploratory phenomenological study in a high case-load country. Int J Environ Res Public Health 18(17):9002. https://doi.org/10.3390/ijerph18179002

Kolozsvári LR, Bérczes T, Hajdu A, Gesztelyi R, Tiba A, Varga I, Al-Tammemi AB et al (2021) Predicting the epidemic curve of the coronavirus (SARS-CoV-2) disease (COVID-19) using artificial intelligence: an application on the first and second waves. Inform Med Unlocked, 25, 100691. https://doi. org/10.1016/j.imu.2021.100691

Lima CK, Tavares PM (2020) The emotional impact of coronavirus 2019-NCoV (New Coronavirus Disease). Psychiatry Res 287:e112915

Lin C-Y (2020) Social Reaction toward the 2019 Novel Coronavirus (COVID-19). Social Health and Behavior 3(1):1-2

Misra S, Le PD, Goldmann E, Yang LH (2020) Psychological impact of anti-asian stigma due to the COVID-19 pandemic: a call for research, practice, and policy responses. Psychol Trauma Theory Res Pract Policy 12(5):461-464

Nicola M, Alsafi Z, Sohrabi C, Kerwan A, Al-Jabir A, Iosifidis C, Agha M, Agha R (2020) The socioeconomic implications of the coronavirus pandemic (COVID-19): a review. Int J Surg 78:185-193

Pantelic M, Boyes M, Cluver L, Meinck F (2017) HIV, Violence, blame and shame: pathways of risk to internalized HIV stigma among south african adolescents living with HIV. J Int AIDS Soc 20(1):21771

Rajkumar RP (2020) COVID-19 and mental health: a review of the existing literature. Asian J Psychiatry 52:102066

Ramaci T, Barattucci M, Ledda C, Rapisarda V (2020) Social Stigma during COVID-19 and Its Impact on HCWs Outcomes. Sustainability 12(9):3834

Ramadan M, Hasan Z, Saleh T, Jaradat M, Al-hazaimeh M, Bani Hani O, Al-Tammemi AB, Shorman E, Al-Mistarehi A-H, Kheirallah K (2021) Beyond knowledge: evaluating the practices and precautionary measures towards COVID-19 amongst medical doctors in Jordan. Inter J Clinical Practice 75(6): 14122

Ransing R, Ramalho R, de Filippis R, Ojeahere MI, Karaliuniene R, Orsolini L, Pinto M, da Costa I, Ullah PG, Bytyçi DG, Grigo O, Mhamunkar A, El Hayek S, Essam L, Larnaout A, Shalbafan M, Nofal M, Soler-Vidal J, Pereira-Sanchez V, Adiukwu F (2020) Infectious disease outbreak related stigma and discrimination during the COVID-19 pandemic: drivers, facilitators, manifestations, and outcomes across the World. Brain Behav Immun 89:555-558

ReedSmith. 2020. "Data Privacy Issues in Connection with the COVID-19 Outbreak: Requirements on Employee Personal Data Differ in China, Hong Kong, and Singapore." [Online]. Retrieved August 1, 2020 (https://www.reedsmith.com/en/perspectives/2020/03/data-privacy-issues-in-connectionwith-the-covid-19-outbreak).

Roberts CM, Gamwell KL, Baudino MN, Grunow JE, Jacobs NJ, Tung J, Gillaspy SR, Hommel KA, Mullins LL, Chaney JM (2020) The contributions of illness stigma, health communication difficulties, and thwarted belongingness to depressive symptoms in youth with inflammatory bowel disease. J Pediatr Psychol 45(1):81-90

Semrau M, Evans-Lacko S, Koschorke M, Ashenafi L, Thornicroft G (2015) Stigma and discrimination related to mental illness in low- and middle-income countries. Epidemiology and Psychiatric Sciences 24(5):382-394 
Taylor S, Landry CA, Rachor GS, Paluszek MM, Asmundson GJG (2020) Fear and Avoidance of Healthcare Workers: An Important, under-Recognized Form of Stigmatization during the COVID-19 Pandemic. J Anxiety Disorders 75:102289

Teksin G, Uluyol OB, Onur OS, Teksin MG, Ozdemir HM (2020) Stigma-Related factors and their effects on health-care workers during COVID-19 pandemics in Turkey: a multicenter study. Sisli Etfal Hastanesi Tip Bulteni 54(3):281-290

Wahed A, Yousif W, Hefzy EM, Ahmed MI, Hamed NS (2020) Assessment of knowledge, attitudes, and perception of health care workers regarding covid-19, a cross-sectional study from Egypt. J Community Health 45(6):1242-1251

Yahya AS, Khawaja S, Chukwuma J (2020) COVID-19 and a potential rise in bullying behaviors. The Primary Care Companion for CNS Disorders 22(3):20com02642

\section{Authors and Affiliations}

\section{Amal Akour ${ }^{1,2}$ (D) Suha A. AlMuhaissen ${ }^{3} \cdot$ Mohammad B. Nusair $^{4}$. Ala'a B. Al-Tammemi ${ }^{5,6,7}$ • Nouf N. Mahmoud ${ }^{2}$ - Sarah Jalouqa ${ }^{3}$. Maissa N. Alrawashdeh ${ }^{8}$}

1 Department of Biopharmaceutics and Clinical Pharmacy, School of Pharmacy, The University of Jordan, Amman 11942, Jordan

2 Department of Pharmacy, Faculty of Pharmacy, Al-Zaytoonah University of Jordan, Amman 11942, Jordan

3 Department of Pharmaceutics and Pharmaceutical Technology, School of Pharmacy, The University of Jordan, Amman, Jordan

4 Department of Clinical Pharmacy and Pharmacy Practice, Faculty of Pharmacy, Yarmouk University, Irbid, Jordan

5 Department of Epidemiology and Global Health, Umeå University, 90187 Umeå, Sweden

6 Department of Family and Occupational Medicine, Faculty of Medicine, University of Debrecen, H-4032 Debrecen, Hungary

7 Doctoral School of Health Sciences, University of Debrecen, H-4032 Debrecen, Hungary

8 Department of Sociology, School of Arts, The University of Jordan, Amman, Jordan 